TITLE:

\title{
A characterization of a limit solution for finite horizon bargaining problems
}

$\operatorname{AUTHOR}(S)$ :

Imai, Haruo; Salonen, Hannu

\section{CITATION:}

Imai, Haruo ... [et al]. A characterization of a limit solution for finite horizon bargaining problems. International Journal of Game Theory 2012, 41(3): 603-622

ISSUE DATE:

2012-08

URL:

http://hdl.handle.net/2433/160135

\section{RIGHT:}

The final publication is available at www.springerlink.com; This is not the published version. Please cite only the published version.; この論文 は出版社版でありません。引用の際には出版社版をご確認ご利用くだ さい。 


\title{
A Characterization of a Limit Solution for Finite Horizon Bargaining Problems
}

\author{
Haruo Imai* Hannu Salonen ${ }^{\dagger}$
}

September 27, 2011

\begin{abstract}
We investigate a two-person random proposer bargaining game with a deadline. A bounded time interval is divided into bargaining periods of equal length and we study the limit of the subgame perfect equilibrium outcomes as the number of bargaining periods goes to infinity while the deadline is kept fixed. This limit is close to the discrete Raiffa solution when the time horizon is very short. If the deadline goes to infinity the limit outcome converges to the time preference Nash solution. Regarding this limit as a bargaining solution under deadline, we provide an axiomatic characterization.
\end{abstract}

Keywords Bargaining, Raiffa solution, Nash solution, Deadline

${ }^{*}$ H. Imai, KIER, Kyoto University, Yoshida-Honmachi, Sakyo, Kyoto, Japan, 606-8501; Tel.: +81-75-753-7113; Fax: +81-75-753-7118; email: imai@kier.kyoto-u.ac.jp

${ }^{\dagger} \mathrm{H}$. Salonen, Department of Economics and PCRC, University of Turku, 20014 Turku, Finland 


\section{Introduction}

We investigate a two-person random proposer bargaining game with a deadline. A bounded time interval is divided into bargaining periods of equal length and we study the limit of the subgame perfect equilibrium outcomes as the number of bargaining periods goes to infinity while the deadline is kept fixed. This limit is close to the discrete Raiffa solution (Raiffa 1951, 1953, 2002) when the time horizon is very short. If the deadline goes to infinity the limit outcome converges to the time preference Nash solution (Chae 1993).

Our interest in finite horizon bargaining problems stems from the observation that many real situations involve fixed deadline. As an example, consider merger negotiations between two firms before special tax benefits for merger expires. Bargaining models with a fixed time horizon are widely used in economic application like incomplete contract theory and search theory. It is also of theoretical interest to study the relation between the Nash bargaining solution and the equilibrium outcome of strategic bargaining models with very little discounting.

In this paper we study first the limit of equilibrium outcomes (as the discount factor goes to one) of a sequential bargaining game under a fixed horizon. After that we explore a class of solutions for dynamic bargaining situations from an axiomatic viewpoint. To do that we have to include in definition of a bargaining problem also parameters corresponding to time horizon and discount factor. To our knowledge, not much effort has been devoted to include the time dimension in the axiomatic bargaining theory (one exception being the recent paper by Diskin, Koppel, and Samet (2009) discussed below).

The sequential bargaining game we analyze is a standard random proposer model, and there is an important precedence of this study by Gomes, Hart, and MasCollel (1999) (referred to as GHM below). In order to investigate the NTU -value of a characteristic function game, they set up a sequential bargaining game (with a probability of breakdown instead of a discount factor), and look at the limit of subgame perfect equilibrium outcomes as the number of period tends to infinity and the breakdown probability vanishes. They find that depending upon the way these two parameters converge, limit outcome could be the Raiffa solution or the Nash solution, and there are borderline cases where the limit outcome lies between these two solutions. The solution studied in our paper corresponds to these borderline cases.

To characterize the limit outcome as the length of a period vanishes, we 
utilize a differential equation obtained by taking the limit process of the continuation values. There are several precursors to this approach. We make use of the proof by Coles and Wright (1998) who study infinite horizon problem with time dependent agreement sets. GHM also use a similar dynamic process to characterize their solution, and they show that the process is related to backwards induction principle. (GHM attribute this method to Maschler, Owen, and Peleg (1988)).

It is well known that with discounting, as the deadline tends to infinity, the subgame perfect equilibrium outcome of the random offer or alternating offer bargaining game converges to the equilibrium outcome of the corresponding infinite horizon game (Binmore 1985). Moreover this outcome converges to the Nash bargaining solution as the discount factor goes to one. All these results are established in Rubinstein (1982) and Binmore (1987). When the deadline is kept fixed, the results change.

Following Stahl (1972), Sjostrom (1991) analyzed a game related to the Rubinstein (1982) model. A finite time interval is divided into bargaining periods of equal length. Sjostrom (1991) proved that without discounting, the subgame perfect equilibrium outcomes converge to the discrete Raiffa solution as the number of bargaining periods increases without limit. He showed also that with very little discounting the limit outcome lies very close to the Raiffa solution. Of course with constant non-negligible discounting this result does not restrict the outcome much and in fact as the deadline goes to infinity, the outcome converges to the Nash solution.

Myerson (1991, p. 393) studies a random proposer, $m$ period bargaining game without discounting. He finds that there is a unique subgame perfect equilibrium for each $m$ and that equilibrium payoffs converge to the discrete Raiffa solution as $m$ goes to infinity. Trockel (2011) analyzes a version of this game such that the first proposer is chosen at random and the responder may either accept it or else choose that they play an $m$ period Myerson game where $m$ is chosen by the responder. Trockel shows that the outcome of each weakly subgame perfect equilibrium is exactly the discrete Raiffa solution.

The discrete Raiffa solution can be obtained as the limit of the following procedure. In the beginning, each agent demands his "ideal point" which is the best outcome for him satisfying individual rationality constraints. Then the average of these two demands are given as a reference point, and players make new demands with the constraint that nobody gets less than this reference point, and so on. The limit outcome of this procedure is the Raiffa solution. Salonen (1988) gives an axiomatic characterization of the Raiffa so- 
lution. For more recent axiomatizations and analysis of the Raiffa solution, see Anbarci and Sun (2009), Diskin et.al. (2009), Samet (2009) and Trockel (2009).

A continuous version of this solution was also proposed by Raiffa and analyzed in Peters and Van Damme(1991) (see also Livne(1989)). Diskin et.al (2009) consider an indexed family of generalized Raiffa solutions that converges to the continuous Raiffa solution. Each member of the family is a pair of functions: a step function that determines a new disagreement point to a bargaining problem and a solution function that assigns the terminal point arrived at. In this way Diskin et.al. (2009) are able to explicitly describe the bargaining process.

Other things that may affect the bargaining outcome except the time dimension are outside options. There are several papers analyzing the problem how the outside alternatives available to the bargainers affect the outcome of the bargaining game (see e.g. Shaked and Sutton (1984) and Binmore, Rubinstein, and Wolinsky (1986)). These studies show that if the best alternative outcome is dominated by the subgame perfect equilibrium outcome, then the best alternative has no effect on the equilibrium outcome. In these papers it is assumed essentially that a player can end the negotiations and take his outside alternative whenever he wants to. However, outside alternatives could matter if this option can be utilized only if no agreement has been reached before a given deadline. ${ }^{1}$

The paper is organized in the following way. The strategic model is analyzed in Section 2. Section 3 is devoted to the axiomatic approach.

\section{The strategic model}

The model we look at is quite similar to standard models in the literature, see e.g. Coles and Wright (1998) where the game has infinite horizon, and GHM where breakdown possibility with risk preference is employed with fixed length of a period. Many of the properties we utilize are established in these papers too.

A 0-1 normalized bargaining problem is given by a compact and convex set $S \subset \mathbb{R}_{+}^{2}$ that contains the origin. The Pareto frontier of $S, P S$, is given

\footnotetext{
${ }^{1}$ It should be noted that we are not dealing with the issues concerning the "deadline effect" (c.f. Fershtman and Seidman 1993; Roth, Murnighan and Schoumaker 1988; Ma and Manove 1993; Simsek and Yildiz 2008).
} 
by $y_{2}=F\left(y_{1}\right)$ such that $1=F(0), 0=F(1)$, where $F$ is concave, continuous, and decreasing. Further, we assume that $F$ and $F^{-1}$ are defined and twice continuously differentiable on some open set $U \supset[0,1]$. For a splitting-a-dollar problem, $F$ is generated by concave, continuous, strictly increasing and twice continuously differentiable functions $u_{1}$ and $u_{2}$ on $[0,1]$ with $u_{i}(0)=0$ and $u_{i}(1)=1(i=1,2)$, such that given $y_{1}$, with $y_{1}=u_{1}(w)$ for some $w \in[0,1], F\left(y_{1}\right)=u_{2}(1-w)$. The Nash solution here is given by $\arg \max _{\left(y_{1}, y_{2}\right) \in S} y_{1} y_{2}=\left\{N^{*}(S)\right\}$.

Given a deadline $T>0$ and a positive integer $m$, let $T / m=\Delta_{m}$ be the length of a period. We count periods backwardly so that $m$ is the first period of the bargaining game. The rules of the sequential bargaining game with a random proposer are as follows. At each period $n$ Nature chooses a proposer with equal probabilities. The chosen proposer makes a proposal $w^{n}$ in $[0,1]$, and another player replies by "Yes" or "No". The number $w^{n}$ is the amount that player 1 would get in case of agreement and then player 2 would get $1-w^{n}$. If the reply is "Yes", then the game ends with a consequence described by $\left(n, w^{n}\right)$. If the reply is "No", then the game moves into period $n-1$ if $n>0$, or ends with payoffs $\left(a_{1}, a_{2}\right)$ (evaluated at $n=0$ ) if $n=0$. We assume that $a=\left(a_{1}, a_{2}\right) \in S$ but unlike Sjostrom (1991), $a=(0,0)$ is not required. Players maximize discounted expected utilities. The discount rate $r>0$ is common to players with the discount factor $\delta=\delta_{r, m}=e^{-r \Delta_{m}}$.

We investigate the subgame perfect equilibrium outcome of this game. To define this concept, we have to define strategies first. The game starts at period $m$ and players get their outside alternatives at the end of period 0 if no agreement is reached prior to that.

We define first histories. Let $\phi^{m}=\emptyset$, and for $n=0, \ldots, m-1$ let $\phi^{n}=\left(i^{t}, w^{t}, \rho^{t}\right)_{t=n+1}^{m}$. The sequence $\left(i^{t}, w^{t}, \rho^{t}\right)_{t=n+1}^{m}$ tells what has happened in previous periods $t \in\{m, \ldots, n+1\}$. In period $t$ player $i^{t}$ was the proposer, $w^{t}$ was the offer he made, and $\rho^{t}=$ "No" was the reply of his opponent. So $\phi^{n}$ is the history in the beginning of period $n$ before the proposer has been selected. Then a period $n$ history for the proposer $i^{n}$ is $\left(\phi^{n}, i^{n}\right)$ and a period $n$ history for the responder is $\left(\phi^{n}, i^{n}, w^{n}\right)$.

A strategy of player $i \in\{1,2\}$ is a mapping $\sigma^{i}$ that maps each period $n$ history for proposer $i$ into $[0,1]$ and each period $n$ history for responder $i$ into \{"Yes","No" $\}$. A strategy profile $\left(\sigma_{1}, \sigma_{2}\right)$ is a subgame perfect equilibrium if conditional on each history, $\sigma_{i}$ is optimal given $\sigma_{j}(j \neq i)$. Let us characterize now the equilibrium outcome. To do that we need to define the (discrete) Raiffa solution with respect to outside option $a=\left(a_{1}, a_{2}\right)$. 
Let $z^{0}=\left(a_{1}, a_{2}\right)$ and $z^{j+1}=\left(\frac{z_{1}^{j}+F^{-1}\left(z_{2}^{j}\right)}{2}, \frac{z_{2}^{j}+F\left(z_{1}^{j}\right)}{2}\right)$ for $j=0,1, \ldots$, and define $R(S, a)=\lim _{j \rightarrow \infty} z^{j}$. As noted by Sjostrom (1991), the Raiffa solution can be defined by $R_{1}(S, a)=\frac{1}{2} \sum_{j=0}^{\infty}\left(F^{-1}\left(z_{2}^{j}\right)-z_{1}^{j}\right)+a_{1}$ and similarly for agent 2 .

If agent 1 is the proposer at the 0 -th period, then he makes an offer $F^{-1}\left(a_{2}\right)$. The corresponding allocation $\left(F^{-1}\left(a_{2}\right), a_{2}\right)$ is accepted by agent 2 . If agent 1 is the responder, then 2 makes an offer $F\left(a_{1}\right)$ which is accepted. This yields the expected payoffs, or the continuation values for period 0 : $\left(z_{1}^{0}, z_{2}^{0}\right)=\left(\frac{1}{2}\left(a_{1}+F^{-1}\left(a_{2}\right)\right), \frac{1}{2}\left(F\left(a_{1}\right)+a_{2}\right)\right)$. For $n \in\{m, \ldots, 1\}$ we have

$$
\left(z_{1}^{n}, z_{2}^{n}\right)=\left(\frac{1}{2}\left(\delta z_{1}^{n-1}+F^{-1}\left(\delta z_{2}^{n-1}\right)\right), \frac{1}{2}\left(F\left(\delta z_{1}^{n-1}\right)+\delta z_{2}^{n-1}\right)\right) .
$$

As usual, an immediate agreement obtains and the subgame perfect equilibrium payoffs are $\left(z_{1}^{m}, z_{2}^{m}\right)$. To evaluate their values in the limit as $\Delta_{m}$ tends to 0 we need some notation. Choose $t \in(0, T]$, and to each $m$ let $n(m)$ be the integer $n \in\{m, \ldots, 1\}$ such that $n T / m$ is closest to $t$. If there are two such integers then let $n(m)$ be the higher one. By construction $\lim _{m \rightarrow \infty} n(m) T / m=t$. We write $z(t)=\lim _{m \rightarrow \infty} z^{n(m)}$, if this limit exists.

Example 1. Suppose that the frontier $P S$ is given by $y_{1}+y_{2}=1$. Writing $z^{n}=\left(z_{1}^{n}, z_{2}^{n}\right)$, (1) becomes $z^{n+1}=\left(\frac{1}{2}\left(\delta z_{1}^{n}+1-\delta z_{2}^{n}\right), \frac{1}{2}\left(1-\delta z_{1}^{n}+\delta z_{2}^{n}\right)\right)$ and if $z^{n}$ lies on the frontier, (which is the case for $n>0$ ) $z^{n+1}-z^{n}=$ $(1-\delta)\left(\frac{1}{2}-z_{1}^{n}, \frac{1}{2}-z_{2}^{n}\right)$. Directly solving it, one obtains for $i=1,2$ that $z_{i}^{n+1}=z_{i}^{n}+(1-\delta)\left(\frac{1}{2}-z_{i}^{n}\right)=\frac{1-\delta}{2}+\delta z_{i}^{n}$. Solving this recursive equation gives us $z_{i}^{n+1}=\delta^{n+1} z_{i}^{0}+\frac{1-\delta^{n+1}}{2}$. Recall that $\delta_{r, m}^{n}=\left(e^{-r T / m}\right)^{n}$, where $T / m=\Delta_{m}$ is the length of the time interval between two consecutive offers. In the limit, as this time interval $\Delta_{m}$ goes to 0 , we have the outcome $z=e^{-r T} R(S, a)+\left(1-e^{-r T}\right) N^{*}(S)$. Note that $N^{*}(S)=(1 / 2,1 / 2)$ and $R(S, a)=z^{0}$ in this case.

We can replace the difference equation above by a differential formula, i.e.

$$
z_{i}^{n+1}-z_{i}^{n}=(1-\delta)\left(\frac{1}{2}-z_{i}^{n}\right)
$$

can be replaced by

$$
\frac{d \log \left(\frac{1}{2}-z_{i}^{t}\right)}{d t}=r
$$


in the limit. This "current value" representation corresponds to the differential equation given by Coles and Wright (1998). It also provides a nice interpretation that the adjustment is made toward the "global" Nash solution $N^{*}(S)$, at a rate proportional to the difference between the current value and Nash solution.

However the present value representation gives also a nice picture. In this example we see that the process jumps from $e^{-r T} a$ to $e^{-r T} R(S, a)$ and from there on it proceeds linearly to $z$ with a direction of $(1 / 2,1 / 2)$.

For a general linear frontier $y_{1} / a+y_{2} / b=1$, we shall have

$$
z^{n}=\left(\delta z_{1}^{n-1}+\frac{a(1-\delta)}{2}, \delta z_{2}^{n-1}+\frac{b(1-\delta)}{2}\right)
$$

or as above,

$$
z^{n+1}-z^{n}=\left((1-\delta)\left(\frac{a}{2}-z_{1}^{n}\right),(1-\delta)\left(\frac{b}{2}-z_{2}^{n}\right)\right)
$$

and

$$
z^{n+1}-z^{n}=(1-\delta)\left(N^{*}(S)-z^{n}\right)
$$

where $N^{*}$ is $(a / 2, b / 2)$. End of Example 1 .

The way differential equations were used in Example 1 turns out to be very convenient in solving the general case as well. In fact Coles and Wright (1998) utilized this approach in their analysis of general random proposer games under non-stationary environments (see also Coles and Muthoo 2003; McLennan 1988; Binmore 1987). We apply their result to the case with a jump in the agreement set at the deadline. For a problem with a smooth Pareto frontier, denote by $f\left(y_{1}\right)$ the derivative $F^{\prime}\left(y_{1}\right), y=\left(y_{1}, y_{2}\right) \in P S$.

Formula (2) allows a specific interpretation, when we employ the notion of a local Nash solution $N(y)$ at a Pareto optimal point $y$. This solution is found by taking the supporting hyperplane of $S$ at $y$ to be the Pareto frontier, and then calculating the usual Nash solution on this plane (the disagreement point being at the origin). In Example 1 the local Nash solution is given by

$$
\left(N_{1}(y), N_{2}(y)\right)=\left(\frac{y_{1}+\frac{y_{2}}{-f\left(y_{1}\right)}}{2}, \frac{-f\left(y_{1}\right) y_{1}+y_{2}}{2}\right) .
$$

The formula (2) then becomes

$$
z^{n+1}-z^{n}=(1-\delta)\left(N\left(z^{n}\right)-z^{n}\right)
$$


Thus the adjustment is made toward the local Nash solution at a rate proportional to the difference, and along the way, the local Nash solution itself moves toward the global Nash solution. We can hope that a similar relation holds approximately for general cases and obtain equivalent of (4) in the limit. We may call the solution of this limit equation as the limit solution or the limit outcome. The following theorem establishes that this limit exists.

Theorem 1 Given $S, a, T$, and $r$ the limit outcome is $x=\left(x_{1}, F\left(x_{1}\right)\right)$ such that

$$
T=\int_{R_{1}(S, a)}^{x_{1}} \frac{1}{r\left(N_{1}\left(z_{1}, F\left(z_{1}\right)\right)-z_{1}\right)} d z_{1}
$$

if $R(S, a) \neq N^{*}(S)$, and $x=N^{*}(S)$ if $R(S, a)=N^{*}(S)$.

Proof. See the Appendix.

Given the limit outcome $x$ in Theorem 1, we describe the solution by its inverse, i.e. as the deadline $T$ needed to reach that particular payoff level. For example, $T=0$ if the payoff corresponds to the Raiffa solution with respect to a. In the proof of the theorem the following lemmata are needed. We denote by $\|\cdot\|$ the sup norm: $\|x\|=\max \left\{\left|x_{1}\right|,\left|x_{2}\right|\right\}$.

Lemma 1 Given $(S, a)$ and $\varepsilon>0$, there is $\bar{\Delta}>0$ and $n$ such that for $\Delta<\bar{\Delta},\left\|R(S, a)-z^{n}\right\|<\varepsilon$ holds, where $z^{n}$ is given by equation (1).

Proof. See the Appendix.

In the following Lemma, $x^{n}\left(y^{n}\right)$ is the equilibrium offer made by player 1 (player 2) in period $n$.

Lemma 2 Given $\varepsilon>0$, there is $\bar{\Delta}$ so that if $\Delta<\bar{\Delta}$, then $\left\|x^{n}-y^{n}\right\|<\varepsilon$ implies $\left\|x^{n+1}-y^{n+1}\right\|<\varepsilon$.

Proof. See the Appendix.

The limit outcome is located between the Nash and the Raiffa solution. A larger $T$ and a lower $r$ moves the solution toward the Nash solution and a higher $a_{i}$ given $a_{j}(i \neq j)$, shifts the Raiffa solution to the advantage of $i$ and hence the solution changes in the same direction. 
As mentioned above, GHM considers a sequential bargaining model with coalition formation based on a characteristic function. In their game, a proposer disappears from the bargaining table with a probability $\rho \in(0,1)$ if his offer is rejected. In case of a pure bargaining problem, this boils down to a sequential bargaining game with a probability of breakdown $\rho$, because as soon as one player disappears from the bargaining table, the surplus to be shared among participants evaporates.

GHM considers the limit payoff configurations of subgame perfect equilibria as $\rho \rightarrow 1$, and the deadline $T \rightarrow \infty$, while keeping the length of a single period fixed at 1 . To characterize their solution, they introduce a dynamic process called Consistent field dynamics $C(y)$, a vector field defined on the Pareto frontier. In this process, each vector $y$ on the Pareto frontier is adjusted toward the consistent value of the corresponding hyperplane game determined by the supporting hyperplanes (Hart and Mas-Colell 1996). They show that this consistent field dynamics is the derivative (times -1 ) of the backward induction function with respect to $\rho$ evaluated at $\rho=1$, for each point $y$ on the Pareto frontier (GHM 1999, Proposition 4.1.).

The consistent field dynamics coincides with the limit dynamics we study above, up to the parameter $r$. But here we let the length of a period vanish, that is, $\Delta_{m} \rightarrow 0$, with $\delta=e^{-r \Delta_{m}}$. Thus by setting $\rho=e^{-h \Delta_{m}}$ where $h$ is an instantaneous rate of breakdown, one sees that changing $\rho$ plays the same role as changing $\Delta_{m}$. By differentiaiting $\rho$ with respect to $\Delta_{m}$ at $\Delta_{m}=0$, their Proposition 4.1 yields that $-C(y)$ equals $-h$ times the time derivative of the backward induction function.

GHM's main interest lies in the case when $T \rightarrow \infty$ faster than $\rho \rightarrow 1$ (or, when $\rho^{T} \rightarrow 0$ ), in which case one obtains the Nash solution in the limit. However, they also note that if $\rho^{T} \rightarrow 1$, then the limit is the Raiffa solution, and in Theorem 4.4 (i) they show that if $\rho^{T} \rightarrow \mu>0$, then the limit can be given by the Consistent field dynamics in the following manner.

Let $\Lambda_{q}(y)$ represent the point reached after duration $q$ when the process starts at $y$, with $\frac{d \Lambda_{q}(y)}{d t}=C\left(\Lambda_{q}(y)\right)$, in a consistent field dynamics. Then the limit point is given by $\Lambda_{-\log \mu}(R(S, a))$. Note that when $\rho=e^{-h T / M}$, then $\rho^{M}=e^{-h T}$ does not depend on $M$ and $e^{-h T}$ corresponds to the term $\mu$ in GHM's Theorem 4.4 (i). (Note that $r$ is a parameter depending upon preferences, while $h$ is exogenously given.) In this sense the limit outcome of our finite horizon game corresponds to the case of GHM explained above. Although these are two different approaches, they both yield a similar formula 
for the solution.

Studying finite horizon problems is of interest in itself, and here we concentrate in this particular case (which GHM does not discuss after Theorem 4.4). The solution in this case depends on the initial point, the Raiffa solution, and the length of time horizon which may indicate that these parameters have some importance in applications as well. This structure of the solution motivates us to study axiomatically a wider class of solutions.

Example 2. For some cases, one can compute the the limit outcome explicitly. Let $u_{1}(w)=w^{\alpha}$ and $u_{2}(w)=w^{\beta}$ with $0<\alpha, \beta \leq 1$ where $w$ is the amount of money in the "divide-a-dollar" problem (these functions are not $C^{1}$ at the boundary, and so $a$ is restricted to the interior of $S$ ). Then

$$
F(u)=\left(1-u^{1 / \alpha}\right)^{\beta} \text { and } \frac{-1}{u+F / F^{\prime}}=\frac{u^{1 /(\alpha-1)}}{\alpha / \beta-(1+\alpha / \beta) u^{1 /(\alpha-1)}}
$$

so that

$$
T\left(x_{1}\right)=-\frac{2}{r(1+\alpha / \beta)} \log \left[\frac{\alpha / \beta-(1+\alpha / \beta) x_{1}^{1 / \alpha}}{\alpha / \beta-(1+\alpha / \beta)\left(R_{1}^{a}\right)^{1 / \alpha}}\right]
$$

or

$$
x_{1}=\frac{\left\{\left[\alpha / \beta-(1+\alpha / \beta)\left(R_{1}^{a}\right)^{1 / \alpha}\right] e^{-(1+\alpha / \beta) r T / 2}\right\}^{\alpha}}{(1+\alpha / \beta)^{\alpha}} \text {. }
$$

A change in the bargaining protocol affects the limit outcome, especially through the change in the limit outcome as $T$ goes to zero. A sequential bargaining game with player $i$ having the last say yields an outcome given by the same formula except that the lower limit of the integral replaced by the $i$-th coordinate of the $i$ 's dictatorial solution instead of that of the Raiffa solution. Under the random proposer protocol with unequal probabilities, the solution is modified according to these probabilities. The Raiffa solution is changed so that the speed of adjustment in the integral is modified, and the local and global Nash solutions are replaced by the asymmetric Nash solutions with the weights given by these probabilities.

\section{The axiomatic model}

The solution given in Theorem 1 is a compound of three elements: 1) the solution reached at the end phase (or the limit when $T$ goes to zero); 2) 
the local adjustment direction for intermediate values of $T$; and 3 ) the limit when $T$ goes to infinity. The particular solutions corresponding to these parts are: 1) the Raiffa solution with respect to the outside alternative; 2) the local Nash solution at each point on the boundary; and 3) the global Nash solution. This motivates us to investigate the relationships between solutions obtained by other possible mixtures of these three elements. For example, the Nash solution is popular for finite horizon problems with outside alternatives, and one may want to construct a strategic model that yields the Nash solution as an equilibrium outcome. The result of the previous section reveals that this might not be an easy task. However, one can still study what kind of axioms are needed to single out the Nash solution for this class of problems.

The class of problems we consider is $\Psi=\left\{B=(S, a, r, T) \mid S \subset \mathbb{R}_{+}^{2}, a \in\right.$ $S$, and $T>0, r>0\}$, where $S$ is a compact, convex and comprehensive subset of $\mathbb{R}^{2}$ such that weakly Pareto optimal elements are Pareto optimal. Thus the class is slightly larger than the one we worked on earlier, where in order to utilize Coles and Wright (1998) result, we assumed smooth Pareto frontiers. Extending the solution given by Theorem 1 to this class is straightforward because the function representing the Pareto frontier of $S$ is differentiable a.e., for all $(S, a, r, T) \in \Psi$, and so the integral defining the solution is still well defined. For a later use, we denote by $\Gamma$ the set of pairs $(S, a)$ such that $B=(S, a, r, T) \in \Psi$, for some $r, T>0$.

We extend the definition of the "derivative" $f$ of the Pareto boundary function $F$ by first defining $f\left(z_{1}\right)=F^{\prime}\left(z_{1}\right)$ if $F$ is differentiable at $z_{1}$. For other values of $z_{1}$ we proceed as follows. Denote by $D$ the set of all points $z_{1}$ at which $F$ is differentiable. Then let $f\left(z_{1}\right)=\lim \left\{f\left(z_{1}^{\prime}\right) \mid z_{1}^{\prime} \uparrow z_{1}, z_{1}^{\prime} \in D\right\}$ if $z_{1}<N_{1}^{*}(S) ; f\left(z_{1}\right)=\lim \left\{f\left(z_{1}^{\prime}\right) \mid z_{1}^{\prime} \downarrow z_{1}, z_{1}^{\prime} \in D\right\}$ if $z_{1}>N_{1}^{*}(S)$; and let $f\left(z_{1}\right)$ be such that $N\left(z_{1}, F\left(z_{1}\right)\right)=N^{*}(S)$ if $z_{1}=N_{1}^{*}(S)$ (recall that $N\left(z_{1}, F\left(z_{1}\right)\right.$ ) is the local Nash solution at $\left(z_{1}, F\left(z_{1}\right)\right)$.

A solution $\varphi$ to bargaining problems in $\Psi$ maps each $B=(S, a, r, T) \in \Psi$ to an element of $S$. We want to axiomatize the solution that selects the limit outcome $x$ given in Theorem 1 . Let us denote this particular solution by $\varphi^{*}$. Consider the following standard properties for solutions on $\Psi$.

Efficiency $(E) . \varphi(B) \in P S$, for all $B \in \Psi$.

Given $\alpha \in \mathbb{R}_{++}^{2}$, denote $\alpha x=\left(\alpha_{1} x_{1}, \alpha_{2} x_{2}\right)$ for all $x \in \mathbb{R}^{2}$. The mapping $\alpha$ defined by $x \longrightarrow \alpha x$ is called a (positive) scale transformation. Given $X \subset \mathbb{R}^{2}$, let $\alpha X=\{\alpha x \mid x \in X\}$. Given $B=(S, a, r, T) \in \Psi$, denote 
$\alpha B=(\alpha S, \alpha a, r, T)$.

Scale Invariance $(S I) . \varphi(\alpha B)=\alpha \varphi(B)$ for each problem $B \in \Psi$, and for any positive scale transformation $\alpha$.

We use the Hausdorff metric on compact subsets of $\mathbb{R}_{+}^{2}$ for measuring the distance between subsets $S$ and $S^{\prime}$ of bargaining problems $B=$ $(S, a, r, T), B^{\prime}=\left(S^{\prime}, a^{\prime}, r^{\prime}, T^{\prime}\right) \in \Psi$. The metric on $\Psi$ is the sup -metric applied to the four "coordinates" $S, a, r, T$ of problems $B \in \Psi$.

Continuity (Cont). $\varphi$ is continuous on $\Psi$.

Denote

$$
G(S, a, r)=\lim _{T \rightarrow 0} \varphi(S, a, r, T) \in S
$$

when the right hand side exists. We call $G(S, a, r)$ the end phase evaluation of $(S, a, r, T)$. By this concept, we try to pin down the bargaining outcome when there is only very little time to bargain. Indeed, continuity implies that the solution in this case is almost $G(S, a, r)$.

If $G(S, a, r)$ does not depend on $r$, then the effect of an outside alternative $a$ is totally captured by $G$, because if two problems share the same future outcomes, then the bargaining outcome today must be the same. Finally, note that combined with the earlier properties, $G$ should be a continuous, efficient, and scale invariant function of $(S, a, r)$.

Time Decomposability $(T D)$. Let $B=(S, a, r, T)$ and $B^{\prime}=\left(S, a, r, T^{\prime}\right)$ with $T>T^{\prime}$. Then $\varphi(B)=\varphi\left(S, \varphi\left(B^{\prime}\right), r, T-T^{\prime}\right)$.

$T D$ states that the solution is decomposable along the time dimension. The problems $B$ and $B^{\prime}$ are otherwise the same except that in $B$ there is more time to bargain $\left(T>T^{\prime}\right)$. Then players could solve $B^{\prime}$ first, and use it's solution as an outside alternative in the new problem where the deadline is at $T-T^{\prime}$.

We are now ready to prove that the end phase evaluation $G(S, a, r)$ exists for every $(S, a, r, T) \in \Psi$, i.e., the limit (5) exists given our axioms. The next Lemma is actually a bit stronger result that will also be used in the proof of Theorem 2 below. We show that $\varphi_{i}(S, a, r, T)$ is either a constant function of $T$, or a strictly monotone function of $T$, or strictly monotone for small values of $T$ and constant elsewhere. Strict monotonicity means that $\varphi_{i}(S, a, r, T)$ is either strictly increasing or strictly decreasing in $T$. 
Lemma 3 Suppose $\varphi$ satisfies $E$, Cont and TD. Then for each problem $B=(S, a, r, T), \varphi_{i}(S, a, r, T)$ is either (i) a constant function of $T$; or (ii) a strictly monotone function of $T$; or (iii) there is a $T^{*}>0$ such that $\varphi$ a strictly monotone function of $T$ on $\left(0, T^{*}\right]$ and a constant function on $\left(T^{*}, \infty\right)$.

Proof. See the Appendix.

Corollary 1 Suppose $\varphi$ satisfies E, Cont and TD. Then the end phase evaluation $G(S, a, r)$ given by (5) exists for all bargaining problems $B=$ $(S, a, r, T) \in \Psi$.

Proof. By Lemma 3, the limit $G(S, a, r)$ defined in equation (5) exists.

By this Corollary, we can extend our class of games $\Psi$ to the class $\bar{\Psi}$ that contains also problems $B=(S, a, r, T)$ with $T=0$, with the understanding that $T=0$ does not imply deadline is immediate, but rather this represents the limit of positive deadline cases. By Corollary 1, we can extend any solution $\varphi$ on $\Psi$ satisfying $E$, Cont and TD to a solution $\bar{\varphi}$ on $\bar{\Psi}$ satisfying these same axioms. However, in order to keep notation simpler, we use the old notation for solutions and the class of problems. Note that the end phase evaluation $G$ is related to $\varphi$ by $\varphi(B)=G(S, a, r)$ for all $B=(S, a, r, 0) \in \Psi$.

Next we modify the independence of irrelevant alternatives axiom (IIA) to our dynamic setting.

Time Path IIA (TPIIA). Let $B=(S, a, r, T) \in \Psi$ and $B^{\prime}=\left(S^{\prime}, a, r, T\right)$ $\in \Psi$ be such that $S^{\prime} \subset S$ and $\varphi(S, a, r, 0)=\varphi\left(S^{\prime}, a, r, 0\right)$. If for all $T^{\prime}, 0<$ $T^{\prime} \leq T, \varphi\left(S, a, r, T^{\prime}\right) \in S^{\prime}$, then $\varphi(B)=\varphi\left(B^{\prime}\right)$.

This property reflects the backward induction principle, and so the justification of our IIA property in the dynamic setting may be more palatable than the justification of the ordinary $I I A$ in static framework.

Next we formulate a symmetry property in our dynamic context. Let $\hat{\pi}$ be the nontrivial permutation on $\{1,2\}$, i.e., $\hat{\pi}(i)=j, i \neq j$. The induced permutation on $\mathbb{R}^{2}$ is denoted by $\pi$ so that $\pi\left(x_{1}, x_{2}\right)=\left(x_{2}, x_{1}\right)$ for any $\left(x_{1}, x_{2}\right) \in \mathbb{R}^{2}$. An element $x \in \mathbb{R}^{2}$ is symmetric if $\pi\left(x_{1}, x_{2}\right)=\left(x_{1}, x_{2}\right)$, and a set $S \subset \mathbb{R}^{2}$ is symmetric if $\pi(S)=\{\pi(x) \mid x \in S\}$ satisfies $\pi(S)=S$. A subset $S \subset \mathbb{R}^{2}$ is symmetric relative to $y \in \mathbb{R}^{2}$, if $S-y$ is symmetric. If a singleton set $\{x\}$ is symmetric relative to $y$, we may say simply that 
$x$ is symmetric relative to $y$. For any $B=(S, a, r, T)$ and $b \in S$, define $S(b)=S \cap\left\{x \in \mathbb{R}_{+}^{2} \mid x \geq b\right\}$.

Dynamic Symmetry $(D S)$. Given $B=(S, a, r, T) \in \Psi$, suppose that for all $t \in(0, T], S\left(e^{-r t} a\right)$ is symmetric relative to $e^{-r T} a$. Then $\varphi(B)$ is symmetric relative to $e^{-r T} a$.

This requirement says that if the "individually rational" part $S\left(e^{-r T} a\right)$ of the problem $B$ is symmetric relative to $e^{-r T} a$, the the solution must be symmetric relative to $e^{-r T} a$. The precondition in $D S$ is rather demanding, but when it is satisfied this axiom becomes very stringent. In fact $D S$ can be applied only to problems with a linear Pareto frontier unless $a_{1}=a_{2}$.

Given a problem $(S, a) \in \Gamma$, the ideal point $M(S, a) \in \mathbb{R}_{+}^{2}$ of $(S, a)$ is defined by $M_{i}(S, a)=\max \left\{y_{i} \mid y \in S, y \geq a\right\}, i=1,2$. We have the following lemma.

Lemma 4 Suppose $\varphi$ is a solution on $\Psi$ satisfying E, SI, Cont, TD and $D S$. If $B=(S, a, r, 0) \in \Psi$ is such that the Pareto set above $a$ is a straight line segment between $\left(M_{1}(S, a), a_{2}\right)$ and $\left(a_{1}, M_{2}(S, a)\right)$, then $\varphi(B)=$ $G(S, a, r)$ is the midpoint of this segment independently of $r$.

Proof. See the Appendix.

Lemma 4 gives sufficient conditions such that if $B=(S, a, r, T)$ is a problem such that the Pareto frontier above $a$ is a straigth line segment, then $\varphi(B)$ is the midpoint of this line independently of $r$. Now any symmetric, efficient and scale invariant bargaining solution on problems $(S, a) \in \Gamma$ has this property.

Here is our second main result.

Theorem 2 Suppose that $\varphi$ satisfies E, SI, Cont, DS, TPIIA and TD on the class $\Psi$ of games. Let $B=(S, a, r, T) \in \Psi$ and let $\varphi(S, a, r, 0)=\left(y_{1}, y_{2}\right)$. Then the solution $\varphi(S, a, r, T)=\left(x_{1}, F\left(x_{1}\right)\right)$ satisfies

$$
T=\int_{y_{1}}^{x_{1}} \frac{1}{r\left\{N_{1}\left(z_{1}, F\left(z_{1}\right)\right)-z_{1}\right\}} d z_{1},
$$

if $y \neq N^{*}(S)$, and $\varphi(S, a, r, T)=y$, if $y=N^{*}(S)$. Further, the end phase evaluation $\varphi(S, a, r, 0)=y$ is independent of $r$. 
Proof. See the Appendix.

By setting $y=R(S, a)$ in Theorem 2 , we see that the limit solution $\varphi^{*}$ satisfies the axioms listed in Theorem 2 . The following proposition holds as well.

Proposition 1 The axioms in Theorem 2 are independent.

Proof. See the Appendix.

In the first part of the paper we noted that if in every bargaining period both players have the same probability of being selected as the proposer, then the "end phase evaluation" would be the Raiffa solution. If the proposer is selected in a different way, then also this solution could change. For example, in the alternating move version of the strategic game, a dictatorial solution would be the end phase evaluation. However, we give here an axiom such that the Raiffa solution results. Consider the following axiom which is adapted from Salonen (1988).

Weak Decomposability $(W D)$. Let $B=(S, a, r, 0), B^{\prime}=\left(S^{\prime}, a, r, 0\right) \in$ $\Psi$ be any two problems such that $S^{\prime} \subset S$ and $M(S, a)=M\left(S^{\prime}, a\right)$. Then there is a problem $B^{\prime \prime}=\left(S^{\prime \prime}, a, r, 0\right)$ with $M\left(S^{\prime \prime}, a\right)=M(S, a)$ and $\varphi\left(B^{\prime \prime}\right)=b$ such that $\varphi(B)=\varphi(S, b, r, 0)$ and $\varphi\left(B^{\prime}\right)=\varphi\left(S^{\prime}, b, r, 0\right)$.

This property says that given any two problems with the same outside alternatives and ideal points, one can find a third problem with the same ideal point and outside option in such a way that the solution of the third problem can be used as a new outside option for the first two problems without changing their solution.

Theorem 3 Let $\varphi$ be a solution on $\Psi$ satisfying $E, S I$, Cont, DS and WD. Then for any $B=(S, a, r, T) \in \Psi, \varphi(S, a, r, 0)=R(S, a)$, that is, the end phase evaluation is the Raiffa solution $R$.

Proof. See the Appendix.

We noted earlier that if we set $y=R(S, a)$ in equation (6), then we get the limit solution $\varphi^{*}$. Hence Theorems 2 and 3 imply the following. 
Corollary 2 The limit solution $\varphi^{*}$ is the only solution on $\Psi$ satisfying axioms E, SI, Cont, DS, TPIIA, TD and WD.

It is easy to show that $W D$ is independent of the other axioms.

We can axiomatize asymmetric versions of $\varphi$ as well. Given the problems $B=(S, a, r, T)$ and $B^{\prime}=\left(S, a^{\prime}, r, T\right)$ we say that $B^{\prime}$ is congruent to $B$, if there is real number $q>0$ such that $S(a)-a=q\left(S\left(a^{\prime}\right)-a^{\prime}\right)$. The real number $q$ is called the scaling factor. Take first the individually rational parts of $B$ and $B^{\prime}$ and translate them so that the least allocation is at the origin. If these sets are related to each other by common scale transformation, then the problems are congruent. Consider the following axiom.

Congruence $(C)$. Suppose all problems $B^{t}=\left(S, e^{-r t} a, r, 0\right)$ are congruent to $B=(S, a, r, 0)$, and $q(t)$ is the scaling factor, $t \in(0, T]$. Then $\varphi(B)-a=q(t)\left(\varphi\left(B^{t}\right)-e^{-r t} a\right)$.

The solutions of congruent problems $B$ and $B^{t}$ are related by the same linear mappings that establishes the congruence.

An asymmetric global Nash solution $N^{* \beta}$ selects from each feasible set $S$ the point that maximizes the product $x_{1}^{\beta} x^{1-\beta}, 0 \leq \beta \leq 1$. Similarly, an asymmetric local Nash solution $N^{\beta}$ at $z$ is defined in the same way as the (symmetric) local Nash solution, except that players have weights $\beta$ and $1-\beta$ in the Nash product. Finally, an asymmetric Raiffa solution $R^{\beta}$ of a problem $(S, a)$ is defined as the limit point of the sequence $\left\{a^{k}\right\}_{k=0}^{\infty}$, where $a^{k+1}=\beta\left(M_{1}\left(S, a^{k}\right), a_{2}^{k}\right)+(1-\beta)\left(a_{1}^{k}, M_{2}\left(S, a^{k}\right)\right.$, and $a_{0}=a$.

One can show that if the axiom $D S$ is replaced by the axiom $C$, then Theorem 2 would hold for a solution $\varphi^{\beta}$ when the global and local Nash solutions are replaced by asymmetric solutions $N^{* \beta}$ and $N^{\beta}$, respectively. Further, with the same replacements, Theorem 3 would hold for the asymmetric Raiffa solution $R^{\beta}$. If in the strategic bargaining game player 1 is chosen as the proposer with probability $\beta$, then the equilibrium outcome would be given by $\varphi^{\beta}$.

We omit detailed proofs because they are simple adaptations of the proofs of Theorems 1 - 3. It suffices to note that when the Pareto frontier of $B=$ $(S, a, r, T)$ is either linear or piecewise linear with only one kink which is proportional to $a$, then $C$ can be applied and it can be shown that, given the axioms, the weight $\beta$ must be the same for all such problems. 


\section{Concluding comments}

We have set out a framework in which parameters representing the dynamic structure of bargaining problems are included in the model and we have characterized a family of solutions axiomatically, in particular the one corresponding to the limit solution of sequential bargaining game with equal recognition probabilities. Like other solutions in the literature, we believe that there are many different ways to characterize these and possibly other, related solutions. Especially the dynamic symmetry axiom we utilized is quite specific and it might be useful to formulate some substitutes for it.

Among possible extensions, one could e.g. increase the number of players. This extension seems rather straightforward, except that the formula representing the solution in two-person problems may not be available for higher dimensions. Another point that may be worth of more careful investigation is how the "end phase "evaluation" changes under different bargaining protocols and how it affects the solution when there is still time to bargain. That might enlarge the set of potential applications of the model.

\section{Acknowledgements}

We are grateful to two anonymous referees for most valuable comments. We thank Bram Driesen, S. Hart, Hans Peters, W. Thomson and J. Urabe for comments and discussions and especially B-G. Ju for the suggestion for systematic treatment in axiomatization. Supports by the Grant-in-Aid for Scientific Research S) 20223001 and B) 20330037 are gratefully acknowledged by Imai.

An earlier version of this paper was written while Salonen was visiting KIER as a JSPS fellow. Salonen is grateful to Japan Society for Promoting Sciences for this fellowship. 


\section{Appendix}

Proof of Lemma 1. We first give names to the mappings defining vectors $z^{n}: G_{0}(z)=\frac{1}{2}\left(z_{1}+F^{-1}\left(z_{2}\right), z_{2}+F\left(z_{1}\right)\right), G(z)=G_{0}(\delta z)$.

Define also $G_{0}^{n}=G_{0} \circ G_{0}^{n-1}$ and $G^{n}=G \circ G^{n-1}$ for $n=1,2, \ldots$, where $G_{0}^{1}=G_{0}$ and $G^{1}=G$. Since $G_{0}$ and $G$ are continuous, so are $G_{0}^{n}$ and $G^{n}$. As $\delta$ tends to $1, G$ tends to $G_{0}$. Therefore $G_{0}^{n}(z)$ tends to $G^{n}(z)$ given $n$ and $z$. Thus given $\varepsilon>0$, there is $\Delta_{m}$ and $n$ so that $\left\|G_{0}^{n}(z)-R(S, a)\right\|<\varepsilon / 2$ and $\left\|G^{n}(z)-G_{0}^{n}(z)\right\|<\varepsilon / 2$. Since $G^{n}(z)=z^{n}$, we have $\left\|R(S, a)-z^{n}\right\|<\varepsilon$, as desired.

Proof of Lemma 2. Let us start by proving the following claim.

Claim. Given $\varepsilon>0$, there is $\bar{\Delta}$ so that if $\Delta<\bar{\Delta}$, then it holds that if $\delta z^{n}$ is $\varepsilon$-close to $P S$, then $\delta z^{n+1}$ is $\varepsilon$-close to PS.

The proof of the Claim. Let $B_{\varepsilon}(P S)$ be an open $\varepsilon$-neighbourhood of the Pareto set $P S$ of $S$ such that $(0,0) \notin B_{\varepsilon}(P S)$. Let $S^{\prime}=S \backslash B_{\varepsilon}(P S)$. Then $S^{\prime}$ is a nonempty compact and convex subset of $S$ such that the distance between $x$ and $P S$ is $\varepsilon$, for every $x \in P S^{\prime}$.

Define the function $G_{0}$ in the same way as in the proof of Lemma 1:

$$
G_{0}(z)=\frac{1}{2}\left(z_{1}+F^{-1}\left(z_{2}\right), z_{2}+F\left(z_{1}\right)\right) .
$$

Choose $\bar{\Delta}>0$ so small that $\delta G_{0}(z) \in B_{\varepsilon}(P S)$ for every $z \in \operatorname{cl}\left[S \backslash S^{\prime}\right]$, where $\delta$ corresponds to $\Delta$, for any $\Delta<\bar{\Delta}$.

To see that such a $\bar{\Delta}$ exists, note first that $\delta G_{0}(z)$ is a continuous function of $\delta$ and $z$ on a compact set $[0,1] \times \operatorname{cl}\left[S \backslash S^{\prime}\right]$. Clearly $\delta G_{0}(z) \in B_{\varepsilon}(P S)$ holds when $\delta=1$. Define

$$
\bar{\delta}=\inf \left\{\delta \in[0,1] \mid \delta G_{0}(z) \in B_{\varepsilon}(P S), z \in c l\left[S \backslash S^{\prime}\right]\right\} .
$$

If $\bar{\delta}=1$, then for any $\delta \in(0,1)$ there is a sequence $\left\{x^{n}\right\}$ such that $\delta G_{0}\left(x^{n}\right) \notin$ $B_{\varepsilon}(P S)$, for all $n$. Since $c l\left[S \backslash S^{\prime}\right]$ is compact, the sequence $\left\{x^{n}\right\}$ has a convergent subsequence and w.l.o.g. we may assume that the sequence itself converges to $x \in \operatorname{cl}\left[S \backslash S^{\prime}\right]$. Then by continuity $\delta G_{0}(x) \notin B_{\varepsilon}(P S)$. But then $x \notin \operatorname{cl}\left[S \backslash S^{\prime}\right]$, since $x<G_{0}(x)$ for every $x \in S \backslash P S$, and $\delta x=\delta G_{0}(x) \in$ $B_{\varepsilon}(P S)$ for some $\delta<1$ when $x \in P S$. Hence $\bar{\delta}<1$, and we may set $\bar{\Delta}$ to correspond $\bar{\delta}$. 
Suppose $z^{n}$ is computed as in equation (1). If $\delta z n \in B_{\varepsilon}(P S)$, then by construction $\delta G_{0}\left(\delta z^{n}\right) \in B_{\varepsilon}(P S)$. Since $\delta G_{0}\left(\delta z^{n}\right)=\delta z^{n+1}$, we are done.

End of the proof of the Claim.

The offers in period $n+1$ are

$$
x^{n+1}=\left(F^{-1}\left(\delta z_{2}^{n}\right), \delta z_{2}^{n}\right) \text { and } y^{n+1}=\left(\delta z_{1}^{n}, F\left(\delta z_{1}^{n}\right)\right) .
$$

The sup -distance between $x^{n+1}$ and $y^{n+1}$ is

$$
d_{s}\left(x^{n+1}, y^{n+1}\right)=\max \left\{F^{-1}\left(\delta z_{2}^{n}\right)-\delta z_{1}^{n}, F\left(\delta z_{1}^{n}\right)-\delta z_{2}^{n}\right\} .
$$

The sup -distance between $\delta z^{n}$ and the Pareto frontier $P S$ is

$$
d_{s}\left(\delta z^{n}, P S\right)=d \text { such that } \delta z^{n}+(d, d) \in P S .
$$

Note that $d_{s}\left(x^{n+1}, y^{n+1}\right) \leq d_{s}\left(\delta z^{n}, P S\right)$. By the Claim, there is $\bar{\Delta}$ so that if $\Delta<\bar{\Delta}$, then it holds that if $\delta z^{n}$ is $\varepsilon$-close to $P S$, then $\delta z^{n+1}$ is $\varepsilon$-close to $P S$. But for this $\bar{\Delta}$ also $d_{s}\left(x^{n+1}, y^{n+1}\right)<\varepsilon$ and $d_{s}\left(x^{n+2}, y^{n+2}\right)<\varepsilon$ so we are done.

Proof of Theorem 1. Note that if $R(S, a)=N^{*}(S)$, then $z^{n+1}-z^{n}=0$ and we obtain the theorem immediately. In the case where $R(S, a) \neq N^{*}(S)$ $N^{*}$, showing that

$$
z_{1}(T)=\int_{0}^{T} r\left[N_{1}\left(z_{1}(t), F\left(z_{1}(t)\right)\right)-z_{1}(t)\right] d t+R_{1}(S, a)
$$

holds is equivalent to prove the theorem.

Given $\varepsilon>0$ and $n$, there is by Lemma $1 \bar{\Delta}^{\prime}$ such that $z^{n}$ is $\varepsilon$-close to $R(S, a)$, when $\Delta<\bar{\Delta}^{\prime}$. By Lemma 2 , we can choose $\bar{\Delta} \leq \bar{\Delta}^{\prime}$ so that if $z^{n}$ is $\varepsilon$-close to $P S$, and hence the offers $x^{n+1}$ and $y^{n+1}$ are $\varepsilon$-close to each other, then the offers $x^{n^{\prime}}$ and $y^{n^{\prime}}$ are $\varepsilon$-close to each other, for all $n^{\prime} \geqq n+1$. Thus Lemmata 1 and 2 in Coles and Wright (1998) hold and the proof of their Theorem 1 applies.

In their Theorem 1, Coles and Wright (1998) show that for the infinite horizon version of this game, the backward induction process satisfies (in the limit as $\Delta \rightarrow 0)$ :

$$
\frac{d q}{d t}=-\frac{r}{2}\left\{\frac{u_{1}}{u_{1}^{\prime}}-\frac{u_{2}}{u_{2}^{\prime}}\right\}
$$


(the sign here is negative since we count time backwards). Recall that $q$ is player 1's monetary gain, player 2 obtains $1-q$, and $u_{i}$ is player $i$ 's utility $(i=1,2)$ with $\frac{d u_{2}(1-q)}{d q}=-u_{2}^{\prime}$. Noting that $y_{1}=u_{1}, F\left(y_{1}\right)=u_{2}$, and $F^{\prime}\left(y_{1}\right)=f\left(y_{1}\right)=-u_{2}^{\prime} / u_{1}^{\prime}$, this formula becomes

$$
-\frac{d y_{1}}{d t}=\frac{r}{2}\left\{F\left(y_{1}\right) / f\left(y_{1}\right)+y_{1}\right\}=r\left[y_{1}-N_{1}(y)\right] .
$$

The rest of the proof is like in the proof of Theorem 1 by Coles and Wright (1998), and the details are omitted here.

Proof of Lemma 3. We show first (Step 1) that if $\varphi$ is a constant function of $T$ on some nonempty interval $\left(T_{1}, T_{2}\right)$, then $\varphi$ is a constant function of $T$ on $\left(T_{1}, \infty\right)$. In this case there exists a least value $T^{*}$ such that $\varphi$ is constant on $\left(T^{*}, \infty\right)$. In Step 2 we show that if $T^{*}>0$ then $\varphi$ is strictly monotone on $\left(0, T^{*}\right]$. If $\varphi$ is not constant on any nonempty interval $\left(T_{1}, T_{2}\right)$, then $\varphi$ is a strictly monotone function of $T$ on $(0, \infty)$.

Step 1. Suppose that $\varphi$ is constant on some $\left(T_{1}, T_{2}\right), T_{1}<T_{2}$, but not everywhere on $\left(T_{1}, \infty\right)$. Then by Cont, there exists a largest $T$ denoted by $\bar{T}$ such that $\varphi$ is constant on $\left(T_{1}, \bar{T}\right)$. Take any $\delta>0, \delta<\bar{T}-T_{1}$ such that $\varphi(S, a, r, \bar{T})=x \neq y=\varphi(S, a, r, \bar{T}+\delta)$. By assumption $\varphi(S, a, r, \bar{T}-\delta)=x$. By $T D, \varphi(S, a, r, \bar{T})=\varphi(S, x, r, \bar{T}-(\bar{T}-\delta))=\varphi(S, x, r, \delta)=x$. But $T D$ implies also that $\varphi(S, a, r, \bar{T}+\delta)=y=\varphi(S, x, r, \bar{T}+\delta-\bar{T})=x$, a contradiction. Hence $\varphi$ must be a constant function of $T$ on $\left(T_{1}, \infty\right)$. By Cont, there exists a least value $T^{*}$ such that $\varphi$ is constant on $\left(T^{*}, \infty\right)$.

Step 2. If $T^{*}>0$, we want to show that $\varphi$ is a strictly monotone function of $T$ on $\left(0, T^{*}\right]$. Assume to the contrary that $\varphi\left(S, a, r, T_{1}\right)=\varphi\left(S, a, r, T_{2}\right)$ for some $T_{1}, T_{2}, 0<T_{1}<T_{2} \leq T^{*}$. For each $\varepsilon>0$ there is $t \in\left(T_{2}-\varepsilon, T_{2}\right)$ such that $\varphi(S, a, r, t) \neq \varphi\left(S, a, r, T_{2}\right)$. This follows by Cont because $\varphi$ is not constant on $\left(T_{2}-\varepsilon, T_{2}\right)$ by Step 1 .

As a continuous function $\varphi_{1}$ achieves a maximum and a minimum value on $\left[T_{1}, T_{2}\right]$. Either the maximum or the minimum value must be different than the value $\varphi_{1}$ gets at $T_{1}$ and $T_{2}$. Suppose w.l.o.g. that $t^{\prime}$ is a maximizer and $\varphi\left(S, a, r, t^{\prime}\right)>\varphi\left(S, a, r, T_{1}\right)=\varphi\left(S, a, r, T_{2}\right)$. Let $\varepsilon$ be such that $2 \varepsilon=$ $\varphi\left(S, a, r, t^{\prime}\right)-\varphi\left(S, a, r, T_{1}\right)$. By Cont, there is a least number $\sigma>0$ such that

$$
\varphi_{1}\left(S, a, r, t^{\prime}-\sigma\right)=\varphi_{1}\left(S, a, r, t^{\prime}\right)-\varepsilon,
$$

and

$$
\varphi_{1}\left(S, a, r, t^{\prime}-\sigma\right)<\varphi_{1}(S, a, r, t)
$$


holds for all $t \in\left(t^{\prime}-\sigma, t^{\prime}\right]$. By Cont, there exists a greatest number $\sigma^{\prime}>0$, $\sigma^{\prime}<T_{2}-t^{\prime}$ such that

$$
\varphi_{1}\left(S, a, r, t^{\prime}+\sigma^{\prime}\right)=\varphi_{1}\left(S, a, r, t^{\prime}\right)-\varepsilon
$$

and

$$
\varphi_{1}\left(S, a, r, t^{\prime}+\sigma^{\prime}\right)>\varphi_{1}(S, a, r, t)
$$

holds for all $t \in\left[t^{\prime}+\sigma^{\prime}, T_{2}\right]$.

Take $\delta>0$ such that $t^{\prime}-\sigma+\delta<t^{\prime}$ and $t^{\prime}+\sigma^{\prime}+\delta<T_{2}$, and let $x=\varphi\left(S, a, r, t^{\prime}-\sigma\right)$. Then by $T D, \varphi\left(S, a, r, t^{\prime}-\sigma+\delta\right)=\varphi(S, x, r, \delta)$, and $\varphi_{1}(S, x, r, \delta)>x_{1}$ by the choice of $\delta$ and $\sigma$. On the other hand, $T D$ implies that $\varphi\left(S, a, r, t^{\prime}+\sigma^{\prime}+\delta\right)=\varphi(S, x, r, \delta)$, and $\varphi_{1}(S, x, r, \delta)<x_{1}$ by the choice of $\delta$ and $\sigma^{\prime}$. This is a contradiction and hence $\varphi\left(S, a, r, T_{1}\right)=\varphi\left(S, a, r, T_{2}\right)$ cannot hold. But then $\varphi$ must be a strictly monotone function on $\left(0, T^{*}\right.$.

If $\varphi$ is not a constant function of $T$ on any nonempty open interval then the argument above shows that $\varphi\left(S, a, r, T_{1}\right)=\varphi\left(S, a, r, T_{2}\right)$ cannot hold for any $T_{1} \neq T_{2}$ and hence $\varphi$ must be strictly monotone on $(0, \infty)$.

Proof of Lemma 4. Suppose first that $a_{i}>0, i=1,2$. Make positive scale transformations to the feasible set $S$ and the outside option $a$ such that for the resulting pair $\left(S^{\prime}, a^{\prime}\right)$ it holds that $M_{1}\left(S^{\prime}, a^{\prime}\right)-a_{1}^{\prime}=M_{2}\left(S^{\prime}, a_{2}^{\prime}\right)-a_{2}^{\prime}$. Denote the transformed game by $B^{\prime}=\left(S^{\prime}, a^{\prime}, 0, r\right)$, and note that by $S I$, the solution $\varphi\left(B^{\prime}\right)$ is obtained from $\varphi(B)$ by applying the same scale transformation that was applied to $B$.

Now $S^{\prime} \cap\left\{x \in \mathbb{R}_{+}^{2} \mid x \geq a^{\prime}\right\}$ is symmetric relative to $e^{-r T} a^{\prime}$, since $T=0$. Then by $D S$ the solution $\varphi\left(B^{\prime}\right)$ is symmetric relative to $a^{\prime}$. But this means that $\varphi_{1}\left(B^{\prime}\right)-a_{1}^{\prime}=\varphi_{2}\left(B^{\prime}\right)-a_{2}^{\prime}$, so $\varphi\left(B^{\prime}\right)$ is the midpoint of the line segment above $a^{\prime}$ that consists of all Pareto optimal points that are above $a^{\prime}$. Since $S$ and $a$ do not depend on $r$, the result holds independently of $r$. When $a_{i}>0$, the result follows by applying $S I$. When $a_{1}=0$ or $a_{2}=0$, the result follows by applying Cont.

Proof of Theorem 2. If $\varphi$ is a constant function of $T$ at $B=(S, a, r, T)$, then the claim clearly holds. Suppose therefore in the remaining proof that $\varphi$ is not a constant function of $T$, and recall that by Lemma 3 that $\varphi$ is then a strictly monotone function of $T$ on $\left(0, T^{*}\right)$ (not excluding the possibility $\left.T^{*}=\infty\right)$ and constant elsewhere.

Suppose first that the Pareto frontier of $B=(S, a, r, T)$ is a straight line segment with a slope -1 . Then the local Nash solution $N\left(z_{1}, F\left(z_{1}\right)\right)$ at $z$ 
is the global Nash solution $N^{*}(S)$. If $y=N^{*}(S)$, then $D S$ implies that $\varphi(B)=N^{*}(S)$. If $y \neq N^{*}(S)$ then formula (6) holds for $\varphi$ by Theorem 1 .

To see this, note first that axioms $E$ and $D S$ imply in this special case that $\varphi(S, a, r, 0)=y$ is the standard symmetric and efficient solution which coincides with the Raiffa solution $R(S, a)$. Secondly, note that we may assume that $w_{1}+w_{2}=1$ holds for Pareto optimal points $w$, so we have that $N\left(z_{1}, F\left(z_{1}\right)\right)=N^{*}(S)=(1 / 2,1 / 2)$. In Example 1 we calculated explicitly that the (first coordinate of the) limit solution in this case is given by $x_{1}=e^{-r T} y_{1}+\left(1-e^{-r T}\right)(1 / 2)$. It suffices to show that formula (6) is satisfied. But integrating the right hand side of (6):

$$
T=\int_{y_{1}}^{x_{1}} \frac{1}{r\left\{N_{1}\left(z_{1}, F\left(z_{1}\right)\right)-z_{1}\right\}} d z_{1}
$$

we get that $r T=-\ln \left(1 / 2-x_{1}\right)+\ln \left(1 / 2+y_{1}\right)$, since $N_{1}\left(z_{1}, F\left(z_{1}\right)\right)=1 / 2$. From this we get easily the correct result $x_{1}=e^{-r T} y_{1}+\left(1-e^{-r T}\right)(1 / 2)$.

If $y=N^{*}(S)$, then $D S$ implies that $\varphi(B)=N^{*}(S)$. If the Pareto frontier is linear but has a different slope than -1 , the result follows from $S I$. Note that the end phase evaluation $\varphi(S, a, r, 0)=y$ does not depend on $r$ and that $\varphi$ is a strictly monotone function of $T$.

Suppose then that the Pareto frontier of $B=(S, a, r, T)$ is piecewise linear. If $\varphi(B)$ and $\varphi(S, a, r, 0)=y$ are on the same line segment, then the result follows from the first part of the proof by TPIIA. Assume next that $\varphi(B)$ and $\varphi(S, a, r, 0)=y$ are on the adjacent line segments $L_{B}$ and $L_{0}$ such that $\varphi(B) \in L_{B} \backslash L_{0}$ and $y \in L_{0} \backslash L_{B}$. To help to visualize the situation, we may assume $\varphi_{1}(B)<y_{1}$.

Then by Cont, we can select $T^{\prime}<T$ in such a way that (i) $\varphi\left(S, a, r, T^{\prime}\right)=$ $x$ is the corner connecting $L_{B}$ and $L_{0}$. Then by $T D, \varphi\left(S, x, r, T-T^{\prime}\right)=$ $\varphi(S, a, r, T)$. Note that if $T^{*}<\infty$, then $T^{\prime}<T^{*}($ since $\varphi(B) \neq x)$ and $\varphi$ is strictly monotone on $\left(0, T^{*}\right]$. If $T^{*}=\infty$ then of course $\varphi$ is strictly monotone.

Since $\varphi$ is strictly monotone on $\left(0, T^{*}\right]$ and $T^{\prime}<T^{*}, T^{\prime}$ is the unique $t<T$ such that $\varphi(S, a, r, t)=x$. Furthermore by strict monotonicity of $\varphi$, $\varphi(S, x, r, t) \neq x$ and $\varphi(S, x, r, t)$ lies between $x$ and $\varphi(B)$ on the line segment $L_{B}$ when $0 \leq t<T-T^{\prime}$.

By TPII $A, \varphi(S, x, r, t)$ is the same as $\varphi\left(S^{\prime}, x, r, t\right)$ for $0 \leq t<T-T^{\prime}$ where the Pareto set of $S^{\prime}$ is the straight line segment $L_{B} \cap \mathbb{R}_{+}^{2}$. By Cont, this holds also for $t=T-T^{\prime}$. 
By TPII A, $\varphi(S, y, r, t)=\varphi\left(S^{\prime \prime}, y, r, t\right)$ for $0 \leq t<T^{\prime}$ where the Pareto set of $S^{\prime \prime}$ is the straight line segment $L_{0} \cap \mathbb{R}_{+}^{2}$. By Cont, the equality holds also for $t=T^{\prime}$. But since $\varphi(S, a, r, t)=\varphi(S, y, r, t)$ holds always when $y=\varphi(S, a, r, 0)$ by Cont and TPIIA, this implies that $\varphi(S, a, r, t)$ is strictly monotone on $\left(0, T^{\prime}\right]$ and hence on $(0, T]$.

In the first part of this proof we showed that formula (6) holds for problems with a linear Pareto frontier. Hence formula (6) holds for $\varphi$ at $\left(S, a, r, T^{\prime}\right)$ and $\left(S, x, r, T-T^{\prime}\right)$ and the end phase evaluations $\varphi(S, a, r, 0)$ and $\varphi(S, x, r, 0)$ of these problems are independent of $r$. Since integration is additive with respect to an integration limit, the result holds. If $\varphi(B)$ and $\varphi(S, a, r, 0)=y$ are not located on adjacent linear segments of a piecewise linear Pareto frontier, then apply induction on the number of corners of line segments between $\varphi(B)$ and $\varphi(S, a, r, 0)=y$.

To any problem $B=(S, a, r, T)$ there is a sequence $\left\{B_{n}\right\}_{n=0}^{\infty}$ of problems having piecewise linear Pareto frontiers converging to $B$. The result follows the from the previous paragraph by Cont.

Proof of Proposition 1. We drop one axiom at a time from Theorem 2 and give a solution that does not satisfy the specifications given in that theorem. Details are left to the reader.

If $E$ is not required, define $\varphi(S, a, T, r)=a e^{-r T}$.

If $S I$ is not required, consider the Egalitarian solution w.r.t. $a$. That is, $\varphi(S, a, T, r)=x \in P S$ such that $x_{1}-a_{1}=x_{2}-a_{2}$.

If $D S$ is not required, define $\varphi(S, a, T, r)=\left(a_{1}, F\left(a_{1}\right)\right)$.

If $T D$ is not required, then let $\varphi(S, a, T, r)$ be the usual Nash solution of the problem $\left(S, e^{-r T} a\right)$, i.e., $S$ is the set of utility allocations and $e^{-r T} a$ is the threat point.

If $C$ ont is not required, then construct a solution as follows. Let $\varphi(S, a, 0, r)=$ $R(S, a)$. If $T>0$, and the Pareto frontier $P S$ is linear in some open neighbourhood around $R(S, a)$, then compute $\varphi(S, a, T, r)$ as in Theorem 2 as long as $\varphi(S, a, T, r)$ is on the same linear segment as $R(S, a)$. If for some $T$ the solution $\varphi(S, a, T, r)$ hits an endpoint of the linear segment containing $R(S, a)$, let $\varphi\left(S, a, T^{\prime}, r\right)$ be equal to that endpoint for all $T^{\prime}>T$. If $R(S, a)$ is not on a linear segment, then let $\varphi(S, a, T, r)=R(S, a)$.

If TPIIA is not required, replace the local Nash solution in the differential equation (6) by the "local Raiffa solution" $R^{l}(z)$ which is defined as follows. As before, $F$ denotes the function $z_{2}=F\left(z_{1}\right)$ describing the Pareto frontier and $R(S,(0,0))$ is the usual Raiffa solution of the bargaining problem 
$(S,(0,0))$. To each point $z$ on the Pareto frontier of the utility set $S$ choose a supporting hyperplane $H(z)$ at $z$ that has the slope equal to a) the right hand side derivative of $F$ at $z_{1}$ if $z_{1}<R_{1}(S,(0,0))$; b) the left hand side derivative of $F$ at $z_{1}$ if $z_{1}>R_{1}(S,(0,0))$; c) the average of the left and right hand side derivatives at $z_{1}$ if $z=R(S,(0,0))$. Let $R^{l}(z)$ be the orthogonal projection of $R(S,(0,0))$ on the plane $H(z)$. Replacing the local Nash solution in equation (6) by the local Raiffa solution gives a solution $\varphi$ on $\Psi$ that satisfies all the other axioms of Theorem 2 except TPIIA

Proof of Theorem 3. Take any $B=\left(S, a^{0}, r, T\right) \in \Psi$, and let $B^{\prime}=$ $\left(S^{\prime}, a^{0}, r, T\right) \in \Psi$ be such that $S^{\prime} \subset S, M\left(S, a^{0}\right)=M\left(S^{\prime}, a^{0}\right)$ and the Pareto set of $B^{\prime}$ above the point $a^{0}$ is a straight line segment. Let the midpoint of this line segment be $a^{1}$, so by Lemma $4, \varphi\left(S^{\prime}, a^{0}, r, 0\right)=a^{1}$. By Lemma 4 and $W D, \varphi\left(S, a^{0}, r, 0\right)=\varphi\left(S, a^{1}, r, 0\right)$, for all $r$.

Suppose that $a^{k}$ has been defined, $a^{k} \geq a^{k-1}$ and that $\varphi\left(S, a^{0}, r, 0\right)=$ $\varphi\left(S, a^{k}, r, 0\right)$, for $k \geq 1$ and independently of $r$. Let $a^{k+1}$ be the midpoint of the line segment between the points $\left(M_{1}\left(S, a^{k}\right), a_{2}^{k}\right)$ and $\left(a_{1}^{k}, M_{2}\left(S, a^{k}\right)\right)$. Then $a^{k+1} \geq a^{k}$ and by $W D$ and Lemma $4, \varphi\left(S, a^{0}, r, 0\right)=\varphi\left(S, a^{k+1}, r, 0\right)$ independently of $r$. Then it follows easily that $\left\{a^{k}\right\}_{k \geq 0}$ is an increasing sequence in $S$ converging to the discrete Raiffa solution $R\left(S, a^{0}\right)$. By construction, $\varphi\left(S, a^{0}, r, 0\right)=R\left(S, a^{0}\right)$ independently of $r$. 


\section{References}

Anbarci N, Sun C (2009) Robustness of intermediate agreements and bargaining solutions. Deakin University, Faculty of Business and Law

Dagan N, Volij O, Winter E (2002) A characterization of the Nash bargaining solution. Soc Choice Welfare 19: 811-23

Diskin AM, Koppel M, Samet D (2009) Generalized Raiffa solutions. Manuscript

Binmore K (1987) Perfect equilibria in bargaining models. In Binmore K, Dasgupta P (eds.) The economics of bargaining. Basil Blackwell, Oxford, pp. $77-105$

Binmore K, Rubinstein A, Wolinsly A (1986) The Nash bargaining solution in economic modelling. Rand J Econ 17: 176-88

Chae S (1993) The n-person Nash bargaining solution with time preference. Econ Letters 41: 21-24

Coles M, Muthoo A (2003) Bargaining in a non-stationary environment. J EconTheory 109: 70-89

Coles M, Wright R (1998) A dynamic equilibrium model of search, bargaining, and money. J Econ Theory 78: 32-54

Fershtman C, and Seidman D (1993) Deadline effects and inefficient delay in bargaining with endogenous commitment. J Econ Theory 60: 306-21

Gomes A, Hart S, Mas-Collel A (1999) Finite horizon bargaining and the consistent field. Games Econ Behav 27: 204-228

Hart S, and Mas-Colell A (1996) Bargaining and value. Econometrica 64: 357-380

Livne ZA (1989) Axiomatic characterization of the Raiffa and the KalaiSmorodinsky solutions to the bargaining problem. Operations Res 37: 972-80 
Ma CA, Manove M (1993) Bargaining with deadlines and imperfect player control. Econometrica 61: 1313-39

Maschler M, Owen G, and Peleg B (1988) Paths leading to the Nash set. In: Roth, AE (ed.) The Shapley Value: Essays in honor of Lloyd Shapley. Cambridge University Press, Cambridge UK, pp. 321-330

McLennan A (1988) Bargaining between two symmetrically informed agents. Mimeo

Myerson RB (1991) Game theory: an analysis of conflict. Harvard University Press, Cambridge, Massachusetts

Nash J (1950) The bargaining problem. Econometrica 18: 155-62

Nash J (1953) Two-person cooperative games. Econometrica 21: 128-40

Peters H, Van Damme E (1991) Characterizing the Nash and Raiffa bargaining solutions by disagreement point axioms. Math Operations Res 16: 447-461

Raiffa H (1951) Arbitration schemes for generalized two person games. University of Michigan

Raiffa H (1953) Arbitration schemes for generalized two-person games. In Kuhn H, Tucker A (eds.) Contributions to the theory of games. Princeton University Press, Princeton, NJ

Raiffa H (2002) Negotiation analysis. Belknap, Harvard

Roth A, Murnighan JK, Schoumaker F (1988) The deadline effect in bargaining: some experimental evidence. Amer Econ Rev 78: 806-23

Rubinstein A (1982) Perfect equilibrium in a bargaining Model. Econometrica 50: $97-110$

Salonen H (1988) Decomposable solutions for n-person bargaining games. 
Europ J Polit Economy 4: 333-347

Samet D (2009) What if Achilles and the tortoise were to bargain? An argument against interim agreements. Manuscript

Shaked A, Sutton J (1984) Involuntary unemployment as a perfect equilibrium in a bargaining mode. Econometrica 52: 1351-64

Simsek A, Yildiz M (2009) Durable bargaining power and stochastic deadlines. Mimeo (March 2009), MIT

Sjostrom T (1991) Stahl's bargaining model. Economic Letters 36: 153-157

Stahl I (1972) Bargaining theory. Stockholm School of Economics

Trockel W (2009) An axiomatization of the sequential Raiffa solution. Institute of Mathematical Economics, University of Bielefeld, Working Papers 425

Trockel W (2011) An exact non-cooperative support for the sequential Raiffa solution. J Math Econ 47: 77-83

Van Damme E (1987) Stability and perfection of Nash equilibria. Springer Verlag, Berlin Heidelberg 